\title{
A THEOREM ON LAPLACE-STIELTJES-INTEGRALS
}

\section{J. MAYER-KALKSCHMIDT}

Let $a(t)$ be a real function which has finite total variation in every finite interval $0 \leqq t \leqq T$. If the Laplace-Stieltjes-Integral

$$
\int_{0}^{\infty} e^{-s t} d a(t)
$$

converges for a finite $s_{0}=x_{0}+i y_{0}$, then there is a region $\operatorname{Re} s>\beta$, where the integral (1) defines an analytic function $f(s)=\mathscr{L}_{s}\{a\}$. The behaviour of this function $f(s)$ on the line $\operatorname{Re} s=\beta$ has been the subject of many investigations (Biggeri [1], Delange [3], Landau [6], Stachó [11]). A basic result is due to Hamburger [5]: The function $f(s)$ has a singularity at $s=\beta$, if $a(t)$ is nondecreasing for all $t \geqq T_{0}$. In this paper we shall extend this theorem to the $(C, k)$-means of (1), for positive integral $k$. (For previous work in this direction see MayerKalkschmidt $[\mathbf{7} ; \mathbf{8}])$.

The $(C, k)$-mean of the integral (1) is defined by the function

$$
m_{k}(s, t)=\frac{1}{t^{k}} \int_{0}^{t}(t-v)^{k} e^{-s v} d a(v) .
$$

\section{If the limit}

$$
\lim _{t \rightarrow \infty} m_{k}(s, t)
$$

exists for a finite $s_{0}$, then it exists in a halfplane $\operatorname{Re} s>\beta_{k}$, the halfplane of $(C, k)$-summability. In this region the limit (3) defines an analytic function $f(s)$, which is the analytic continuation of $f(s)$ $=\mathscr{L}_{s}\{a\}$, if $\beta_{k}<\beta$. For proofs of these and further theorems in the theory of the $(C, k)$-means the reader is referred to Bosanquet [2], Doetsch [4], McCrossen [10]. The following theorem is analogous to Hamburger's theorem. ${ }^{1}$

Theorem A. Let $\beta_{k}$ be the abscissa of $(C, k)$-summability of the integral (1) and let $f(s)$ be the function defined by this integral. If

$$
m_{k}\left(\beta_{k}, t\right) \text { is nondecreasing for } t \geqq T_{0} \geqq 0 \text {, }
$$

and if

Received by the editors June 23, 1958 and, in revised form, August 18, 1958. $[8]$.

1 This theorem represents the correction of Theorem 3.1 in the author's paper 


$$
f^{(r)}\left(\beta_{k}+\right)=0 \quad \text { for } r=1, \cdots, k,
$$

then $f(s)$ has a singularity ${ }^{2}$ at $s=\beta_{k}$.

The proof will indicate that conditions (4) and (5) are, in a sense, necessary.

For the proof we need two lemmas which are interesting in themselves.

Let $[L, c, n]$ stand for the class of functions $g(s)$ which satisfy the following conditions:

(a) $g(s)$ is analytic at $s=0$,

(b) $g(s)$ can be represented in $\operatorname{Re} s>0$ by a Laplace-StieltjesIntegral,

(c) $g^{(r)}(0)=c_{r}, r=1, \cdots, n$.

Lemma 1. Let $A_{a}^{p}$ stand for the binomial coefficient $C_{p}^{p+q}$, denote $d_{r}=(-1)^{r} c_{r} / r !$ and $x_{m}=\operatorname{det} a_{i k}$, where $a_{i k}=A_{i}^{k-1}$ for $i=1, \cdots, n$ and $k=1, \cdots, m-1, m+1, \cdots, n$ and $a_{i k}=d_{i}$ for $k=m$. Then the function

$$
g(s)=\sum_{m=1}^{n} \frac{x_{m}}{(1+s)^{m}}
$$

belongs to the class $[L, c, n]$. The $(C, k)$-mean of (6) has for $k \leqq n$ at $s=0$ the form ${ }^{3}$

$$
m_{k}(g, 0, t)=c_{0}+\sum_{p=1}^{k} \frac{C_{p}^{k} c_{p}}{t^{p}}-e^{-t} P(t),
$$

where $c_{0}=\sum_{m=1}^{n} x_{m}$, and

$$
P(t)=(-1)^{k} \sum_{m=1}^{n} \frac{x_{m}}{(m-1) !} \sum_{p=k}^{m+k-1} p ! C_{p-k}^{m-1} t^{m-1+p} .
$$

A similar formula has been obtained by Doetsch [4, p. 341$]$.

Proof. It is obvious that the function (6) is analytic at $s=0$. Condition (b) is also satisfied, because of the formula

$$
\frac{(m-1) !}{(1+s)^{m}}=\int_{0}^{\infty} e^{-s t} e^{-t} t^{m-1} d t .
$$

If the sum in (6) is expanded in a powerseries in $s$

2 The $r$ th right derivative of the function $f(s)$ at the point $s_{0}$ is defined by the limit $f^{(r)}\left(s_{0}+\right)=\lim _{s_{\rightarrow} s_{0}+}\left(f^{(r-1)}(s)-f^{(r-1)}\left(s_{0}+\right)\right) /\left(s-s_{0}\right)$.

3 In general $m_{k}(h, s, t)$ will denote the $(C, k)$-mean constructed from the function $h(s)$; if $h(s)=f(s)$, we shall write $m_{k}(f, s, t)=m_{k}(s, t)$. 


$$
\sum_{m=1}^{n} \frac{x_{m}}{(1+s)^{m}}=\sum_{m=1}^{n} x_{m} \sum_{p=0}^{\infty}(-s)^{p} A_{p}^{m-1}=\sum_{p=0}^{\infty}(-s)^{p} \sum_{m=1}^{n} A_{p}^{m-1} x_{m},
$$

it is seen that the first $n$ derivatives of $g(s)$ have, for $s=0$, the value

$$
a_{r}=(-1)^{r} r ! \sum_{m=1}^{n} A_{r}^{m-1} x_{m}=(-1)^{r} r ! \sum_{m=1}^{n} A_{r}^{m-1} \sum_{p=1}^{n}(-1)^{m+p} d_{p} A_{p m},
$$

where $r=1, \cdots, n$, and where $A_{p m}$ is the subdeterminant constructed from $\operatorname{det} A_{i}^{\boldsymbol{k}},(i=1, \cdots, n ; k=0, \cdots, n-1)$, by deleting the $p$ th row and the $m$ th column. Changing the order of summation in (9) yields

$$
a_{r}=r ! \sum_{p=1}^{n}(-1)^{p} d_{p} \sum_{m=1}^{n}(-1)^{m+r} A_{r}^{m-1} A_{p m}=(-1)^{r} r ! d_{r}=c_{r}, r=1, \cdots, n
$$

which proves the first part of the lemma.

For the proof of the second part we observe, that equations (6) and (8) imply the equation

$$
g(s)=\int_{0}^{\infty} e^{-s t} \sum_{m=1}^{n} \frac{x_{m}}{(m-1) !} e^{-t} t^{m-1} d t .
$$

Therefore

$$
m_{k}(g, 0, t)=\sum_{m=1}^{n} \frac{x_{m}}{(m-1) !} \frac{1}{t^{k}} \int_{0}^{t} e^{-v}(t-v)^{k} v^{m-1} d v .
$$

Applying the Binomial Theorem we obtain

$$
m_{k}(g, 0, t)=\sum_{m=1}^{n} \frac{x_{m}}{(m-1) !} \frac{1}{t^{k}} \sum_{r=0}^{k} C_{r}^{k} t^{k-r}(-1)^{r} \int_{0}^{t} e^{-v} v^{m-1+r} d v .
$$

Integration by parts yields

$$
\begin{aligned}
m_{k}(g, 0, t)= & \sum_{m=1}^{n} \frac{x_{m}}{(m-1) !} \sum_{r=0}^{k} C_{r}^{k} t^{-r}(-1)^{r} \\
& \cdot\left\{(m-1+r) !-e^{-t} \sum_{p=0}^{m-1+r} t^{m-1+r-p} \frac{(m-1+r) !}{(m-1+r-p) !}\right\} .
\end{aligned}
$$

We invert the order of summation and find

$$
m_{k}(g, 0, t)=\sum_{r=0}^{k} C_{r}^{k} t^{-r}(-1)^{r} r ! \sum_{m=1}^{n} x_{m} \frac{(m-1+r) !}{(m-1) ! r !}-e^{-t} P(t) .
$$

This reduces to 


$$
m_{k}(g, 0, t)=\sum_{r=0}^{k} \frac{C_{r}^{k} c_{r}}{t^{r}}-e^{-t} P(t)
$$

where

$$
P(t)=\sum_{m=1}^{n} \frac{x_{m}}{(m-1) !} \sum_{p=0}^{m-1+k} t^{m-1-p} \sum_{r=p+m-1}^{m-1+k} C_{r}^{k}(-1)^{r} \frac{(m-1-r) !}{(m-1-p+r) !} \cdot
$$

Since $C_{r}^{k}=0$ for $r>k$, the inner sum shrinks to

$$
\sum_{r=0}^{k} C_{r}^{k}(-1)^{r}(m-1+r) \cdots(m-1+r-p+1)
$$

and this expression is equal to the $p$ th derivative of the function $(1-t)^{k} t^{m-1}$ for $t=1$. This value is zero for $p<k$. For $p=k$ we have the value $(-1)^{k} k$ !, and for $p>k$ we obtain by applying Leibnitz' rule to the mentioned function the value $C_{\boldsymbol{k}}^{p}(-1)^{k} k !(m-1) \cdots(m+k-p)$. This gives us the stated formula for $P(t)$, and the proof of Lemma 1 is complete.

Lemma 2. Let $k$ be an integer. Then the statements

$$
f^{\prime}\left(\beta_{k}+\right)=f^{\prime \prime}\left(\beta_{k}+\right)=\cdots=f^{(n)}\left(\beta_{k}+\right)=0, f^{(n+1)}\left(\beta_{k}+\right)>0,
$$

and

$$
m_{k}\left(\beta_{k}, t\right) \quad \text { is nondecreasing for } t \geqq T_{0} \geqq 0,
$$

contradict each other for all integral $n<k$.

For the corresponding theorem on power series see Mayer-Kalkschmidt [9].

PROOF. Without restriction of generality we can assume $\beta_{k}=0$. We show first that $f^{\prime}(0+)>0$ and (11) are contradictory. The function

$$
y_{k}(0, t)=\frac{k}{t^{k+1}} \int_{0}^{t}(t-v)^{k-1} v d a(v)
$$

exists almost everywhere, and almost everywhere the equation $d m_{k}(0, t) / d t=y_{k}(0, t)$ holds. Furthermore we have for the derivative of the function $f(s)$ the formula

$$
f^{\prime}(s)=-\int_{0}^{\infty} e^{-s t} t d a(t),
$$

which, for $\operatorname{Re} s>0$, can be represented in the form 


$$
f^{\prime}(s)=-\frac{s^{k}}{(k-1) !} \int_{0}^{\infty} e^{-s t} \int_{0}^{t}(t-v)^{k-1} v d a(v) d t .
$$

Condition (11) implies that for positive $s$ we have

$$
f^{\prime}(s) \leqq-\frac{s^{k}}{(k-1) !} \int_{0}^{T_{0}} e^{-s t} \int_{0}^{t}(t-v)^{k-1} v d a(v) d t,
$$

and the right side tends to zero as $s$ approaches the origin. Hence, if $f^{\prime}(0+)$ exists, we have the inequality

$$
f^{\prime}(0+)=\lim _{s \rightarrow+0} \frac{f(s)-f(0)}{s} \leqq \lim _{s \rightarrow+0 ; 0<z<s} f^{\prime}(z) \leqq 0,
$$

which implies the desired contradiction.

In order to complete the proof of the lemma we construct a function $h(s)$ such that

$$
\frac{f^{\prime}(s)}{s^{n}}=h^{\prime}(s)
$$

for $\operatorname{Re} s>0$, where $n$ is a positive integer. If this function has the representation

$$
h(s)=\int_{0}^{\infty} e^{-s t} d b(t),
$$

we find from equation (12) the relation

$$
-\int_{0}^{\infty} e^{-s t} t d b(t)=-C_{1} \int_{0}^{\infty} e^{-s t} \int_{0}^{t}(t-v)^{n-1} v d a(v) d t .
$$

Because of the uniqueness of the Laplace-Stieltjes-Transform this implies

$$
\int_{0}^{t}(t-v)^{q-1} v d b(v)=C_{2} \int_{0}^{t}(t-v)^{n+q-1} v d a(v),
$$

where $q$ is a positive integer and $C_{2}$ is a positive constant. Now, let $y_{k}(h, 0, t)$ correspond to $(13)$ as $y_{k}(0, t)$ corresponds to (1). Then an implication of equation (14) is that

implies

$$
y_{n+q}(0, t) \leqq 0 \text { for } t \geqq T_{0} \geqq 0
$$

$$
y_{q}(h, 0, t) \geqq 0 \text { for } t \geqq T_{0} \geqq 0 .
$$

If we multiply equation (12) by $s^{n}$, differentiate it $n$ times we obtain 


$$
f^{(n+1)}(s)=\sum_{m=0}^{n} C_{m}^{n}\left(s^{n}\right)^{(m)} h^{(n+1-m)}(s) .
$$

Because of condition (10) this implies that $\lim _{s \rightarrow+0} f^{(n+1)}(s)$ $=C_{3} \lim _{8 \rightarrow+0} h^{\prime}(s)$, where $C_{3}$ is a positive constant. Hence, with $n+q=k$, the following implications are true

$$
f^{(n+1)}(0+) \geqq 0 \quad \text { if and only if } h^{\prime}(0+) \geqq 0,
$$

and

$$
y_{k}(0, t) \geqq 0 \quad \text { if and only if } y_{k}(h, 0, t) \geqq 0 .
$$

The two statements on the right contradict each other, therefore the same is true for the left hand statements, and the lemma is proved.

Now we proceed to prove Theorem A. We assume without restriction of generality that $\beta_{k}=0$. According to the theorem by Hamburger, condition (4) implies that the Laplace-Stieltjes-Integral

$$
\int_{0}^{\infty} e^{-s t} d m_{k}(0, t)
$$

defines a function $F(s)$ which has a singularity for $s=\boldsymbol{\gamma}$, where $\boldsymbol{\gamma}$ is the abscissa of convergence of the integral (15). We show that this property transfers to the function $f(s)$.

Since $(1)$ is $(C, k)$-summable, $\gamma \leqq 0$. Then, by integrating the integral (15) by parts, we obtain for $\operatorname{Re} s>0$ the relation

$$
\frac{F(s)}{s}=\int_{0}^{\infty} e^{-s t} m_{k}(0, t) d t .
$$

Differentiating this expression $k$ times, we find

$$
\left(\frac{F(s)}{s}\right)^{(k)}=(-1)^{k} \int_{0}^{\infty} e^{-8 t t^{k}} m_{k}(0, t) d t
$$

and finally, by an elementary argument,

$$
\left(\frac{F(s)}{s}\right)^{(k)}=(-1)^{k} k ! \frac{f(s)}{s^{k+1}} .
$$

This equation, together with condition (4) and Hamburger's theorem implies that $\gamma \geqq 0$. Therefore $\gamma=0$, and $F(s)$ has a singularity at $s=0$. The same is true for $f(s)$ as the following indirect proof shows.

Let us assume that the function $f(s)$ is analytic at $s=0$, and that the power series expansion is, according to condition (5),

$$
f(s)=c_{0}+c_{k+1} s^{k+1}+c_{k+2} s^{k+2}+\cdots .
$$


If this expression is substituted in equation (16), and this equation is then integrated $k$ times, along a path in the right half-plane, one obtains the expansion

$$
F(s)=a_{0}+\sum_{n=1}^{k} a_{n} s^{n}+(-1)^{k} k \sum_{n=k+1}^{\infty} c_{n} B(n-k, k) s^{n},
$$

where the $a_{n}$ are constants of integration, and $B(p, q)$ is the Euler integral of first kind. Hence we have found, that if $f(s)$ is analytic at $s=0$, and has the expansion (17), then $F(s)$ is also analytic at $s=0$.

Now we show, that condition (5) is necessary for the truth of the theorem. For this we assume again, that $f(s)$ is analytic, but we let $f(s)$ have the expansion $f(s)=c_{0}+c_{1} s+c_{2} s^{2}+\cdots$, where at least one of the $c_{n}, 1 \leqq n \leqq k$, does not vanish. If we insert this expansion in equation (16) and integrate as before, at one step we shall introduce a $\operatorname{logarithm}$, and we can say nothing about the analyticity of $F(s)$. Now we construct the function $h(s)=f(s)-g(s)$, where $g(s)$ is the function constructed in Lemma 1 (for the case $n=k$ ). The function $h(s)$ is then analytic at $s=0$, and has an expansion like (17). Hence we can repeat the argument above, if only the $(C, k)$-mean corresponding to the function $h(s), m_{k}(h, 0, t)$, increases monotonically for $t \geqq T_{0} \geqq 0$. According to Lemma 1 we have

$$
m_{k}(h, 0, t)=m_{k}(0, t)-\sum_{p=0}^{k} \frac{C_{p}^{k} c_{p}}{t^{p}}+e^{-t} P(t),
$$

and this function increases monotonically for large $t$, if the first nonzero $c_{p}, p \geqq 1$, is positive. However Lemma 2 shows, that this contradicts condition (4). This indicates in which sense condition (5) is necessary.

\section{REFERENCES}

1. C. Biggeri, Sur les singularités des intégrales de Laplace, C. R. Acad. Sci. Paris vol. 205 (1937) pp. 600-603.

2. L. S. Bosanquet, The summability of Laplace-Stieltjes-Integrals, Proc. London Math. Soc. (3) vol. 3 (1953) pp. 267-304.

3. H. Delange, Sur les singularités des intégrales des Laplace, C. R. Acad. Sci. Paris vol. 233 (1951) pp. 1413-1414.

4. G. Doetsch, Handbuch der Laplace-Transformation I, Basel, 1950.

5. H. Hamburger, Bemerkungen zu einer Fragestellung des Herrn Poblya, Math. Z. vol. 7 (1920) pp. 302-322.

6. E. Landau, Über einen Satz von Tschebyscheff, Math. Ann. vol. 61 (1905) pp. 527-550.

7. J. Mayer-Kalkschmidt, Singularitäten von Laplace-Integralen an der Summierbarkeitsabszisse, Arch. Math. vol. 4 (1953) pp. 441-445. 
8. - Zur Theorie der Laplace-Stieltjes-Integrale, Mitt. Math. Sem. Giessen vol. $47,1954,26$ pages.

9. - Über Singularitäten gewisser Potenzreihen, Arch. Math. vol. 7 (1956) pp. 129-134.

10. G. McCrossen, A generalized Laplace-Stieltjes-Transformation, Proc. Amer. Math. Soc. vol. 8 (1957) pp. 278-285.

11. T. von Stach6, Randsingularität Laplacescher Transformierten, Monatsh. Math. Phys. vol. 48 (1939) pp. 408-418.

University of New Mexico 